\title{
A Note on the Malliavin differentiability of the Heston Volatility
}

\author{
Elisa $\operatorname{Alos}^{1}$, Christian-Oliver Ewald ${ }^{2}$ \\ ${ }^{1}$ Department of Economics, University of Pompeu Fabra, c/Ramon Trias \\ fargas, 25-27, 08005 Barcelona, Spain ( e-mail : elisa.alos@upf.edu ) \\ 2 School of Mathematics, Woodhouse Lane, University of Leeds, LS2 9JT, \\ Leeds, UK ( e-mail : ewald@maths.leeds.ac.uk )
}

\begin{abstract}
We show that the Heston volatility or equivalently the Cox-IngersollRoss process satisfying

$$
d v_{t}=\kappa\left(\theta-v_{t}\right) d t+\nu \sqrt{v_{t}} d W_{t}
$$

is Malliavin differentiable and give an explicit expression for the derivative. This result assures the applicability of Malliavin calculus in the framework of the Heston stochastic volatility model and the CoxIngersoll-Ross model for interest rates.
\end{abstract}

Keywords: Malliavin calculus, stochastic volatility models, Heston model, Cox-Ingersoll-Ross process

AMS Subject Classification: $\quad 60 \mathrm{H} 07,65 \mathrm{H} 10,65 \mathrm{H} 30,91 \mathrm{~B} 28,91 \mathrm{~B} 70$

JEL Subject Classification: G12, G19, C19, E43 


\section{Introduction}

In recent years, Malliavin calculus has appeared as a major tool in both theoretical and computational mathematical finance. This fact is document by the large amount of published work in this area, see [1], [3], [11], [16], [19] for contributions on the theoretical aspects and [2],[4], [5],[6], [8],[10], [12], [13], [14] for computational aspects. In all these publications, the assumption on the possibly multidimensional diffusion process $\left(X_{t}\right)$ which determines the factors of the model, are very strict. In fact, the classical results assume that the coefficient functions $\beta$ and $\sigma$ in

$$
d X_{t}=\beta\left(X_{t}, t\right) d t+\sigma\left(X_{t}, t\right) d \mathbb{W}_{t}
$$

have bounded derivatives of all orders and have linear growth at infinity. In some publications the assumption are even more strict. These assumptions work fine with the standard Black-Scholes model. Problems occur however when one uses more advanced models, like the Heston stochastic volatility model. In this model the stock price is given by the equation

$$
d S_{t}=S_{t}\left(b d t+\sqrt{v_{t}} d B_{t}\right)
$$

where $\left(B_{t}\right)$ denotes a Brownian motion but in contrast to the standard BlackScholes model the volatility $v_{t}$ is itself a diffusion process, satisfying the stochastic differential equation

$$
d v_{t}=\kappa\left(\theta-v_{t}\right) d t+\nu \sqrt{v_{t}} d W_{t}
$$

where $W_{t}$ denotes a possibly correlated second Brownian motion. As one can see on first glance, the coefficient functions of this model do not satisfy the standard assumptions. The square root function does not have bounded derivatives. However the Heston stochastic volatility model is one of the most popular among the stochastic volatility models, both from the theoretical and empirical point of view. Malliavin calculus in the framework of the Heston stochastic volatility model has been considered in [10], but in this article a direct application of the Malliavin derivative operator on the Heston volatility has been avoided. Among the experts there was widely speculation about whether the Heston volatility is Malliavin differentiable. In this article we give an answer to this longstanding and significant question. We give a direct proof of the Malliavin differentiability of the Heston volatility and its square root and give explicit expressions for their derivatives. This is a key result, as it justifies the applicability of Malliavin calculus in the framework of the Heston stochastic volatility model and the Cox-Ingersoll-Ross interest rate model. Our result turns out to be a powerful tool, many application can 
be found in the literature mentioned in the beginning of this introduction. Given our result, these application can now be directly adapted to the Heston stochastic volatility model. This is the reason why we omit any explicit application in this article. The same process as in equation (2) but classically denoted with $r_{t}$ rather than $v_{t}$ has been used in interest rate theory in the framework of the famous Cox-Ingersoll-Ross model [7]. Our results therefore directly apply to this model too. However, here we leave this by side. In our proof we use a result by Detemple et al. [9] which allows to represent the Malliavin derivative of a diffusion in an elegant and computational advantageous way. Though Detemple's result also uses strict assumptions such as boundedness of all derivatives of the coefficient functions, we are able to generalize Detemple's formula for the case of the Heston volatility.

\section{The Heston volatility model and an ap- proximating sequence}

As mentioned in the introduction, the Heston stochastic volatility model consists of a bond, which we do not specify in this article, a stock $\left(S_{t}\right)$ and the volatility process $\left(v_{t}\right)$ with dynamics specified in (1) and (2) in the introduction. It is assumed that $\kappa, \theta$ and $\nu$ are positive constants. This model was first used by Heston in [15]. Heston computed explicit formulas for the value of European calls in this model. In the following we consider one fixed probability space $(\Omega, \mathcal{F}, \mathbb{P})$ on which there is defined a Brownian motion $\left(W_{t}\right)$ and which is filtered by the augmented and completed Brownian filtration. We also fix an interval $[0, T]$. A standard assumption, when using the Heston model is

$$
2 \kappa \theta \geq \nu^{2} .
$$

This is often called the Novikov condition. Given that $v_{0}>0$ this condition guarantees that the volatility process is always positive, i.e.

$$
\mathbb{P}\left(\left\{v_{t}>0 \forall t>0\right\}\right)=1 .
$$

We assume that $v_{0}>0$ and that the Novikov condition holds. It is then possible to consider the square root process $\sigma_{t}:=\sqrt{v_{t}}$. It follows from the Itô formula that this process satisfies

$$
d \sigma_{t}=\left(\frac{\kappa \theta}{2}-\frac{\nu^{2}}{8}\right) \frac{1}{\sigma_{t}}-\frac{\kappa}{2} \sigma_{t}+\frac{\nu}{2} d W_{t}
$$


We note that the Novikov condition implies in particular that the factor $\left(\frac{\kappa \theta}{2}-\frac{\nu^{2}}{8}\right)$ appearing in the drift term of $\sigma_{t}$ is positive. This will play a role later. We will need the following Lemma to compare processes and to apply the dominated convergence theorem in our approximation of the process $\sigma_{t}$. The Lemma is a special case of the Comparison Lemma as it can be found in [17], Chapter 5, Proposition 2.18.

Lemma 2.1. Consider the two Itô processes

$$
\begin{aligned}
& X_{t}^{1}=X_{0}+\int_{0}^{t} \beta_{1}\left(X_{s}^{1}\right) d s+k W_{t}, \\
& X_{t}^{2}=X_{0}+\int_{0}^{t} \beta_{2}\left(X_{s}^{2}\right) d s+k W_{t},
\end{aligned}
$$

where $\beta_{1}, \beta_{2}: \mathbb{R} \rightarrow \mathbb{R}$ are two measurable functions and $k$ is a positive constant. Assume that (6) and (7) have a strong solution and suppose that $\beta_{1} \geq$ $\beta_{2}$ and at least one of them satisfies a Lipschitz condition. Then $X_{t}^{1} \geq X_{t}^{2}$ for all $t$, a.s.

In order to show in section 4 , that $\sigma_{t}$ is Malliavin differentiable we now define an approximating sequence. This sequence shows to be useful in other aspects of the Heston model as well. Let $\varepsilon>0$ and $\Phi_{\varepsilon}(x)$ be a continuously differentiable function satisfying

$$
\Phi_{\varepsilon}(x)= \begin{cases}1 & \text { if } \quad x \geq 2 \varepsilon \\ 0 & \text { if } \quad x<\varepsilon\end{cases}
$$

as well as $\Phi_{\varepsilon}(x) \leq 1$ for all $x \in \mathbb{R}$. We note that

$$
\Phi_{\varepsilon}^{\prime}(x)= \begin{cases}0 & \text { if } \quad x \geq 2 \varepsilon \\ 0 & \text { if } \quad x<\varepsilon\end{cases}
$$

Furthermore we define the function

$$
\Lambda_{\varepsilon}(x)=\Phi_{\varepsilon}(x) \frac{1}{x}
$$

with $\Lambda_{\varepsilon}(0)=0$. The function $\Lambda_{\varepsilon}(x)$ is bounded and continuously differentiable with

$$
\Lambda_{\varepsilon}^{\prime}(x)=\Phi_{\varepsilon}^{\prime}(x) \frac{1}{x}-\Phi_{\varepsilon}(x) \frac{1}{x^{2}}=\left\{\begin{array}{lll}
-\frac{1}{x^{2}} & \text { if } & x \geq 2 \varepsilon \\
0 & \text { if } \quad x<\varepsilon
\end{array}\right.
$$


Let us now define our approximations $\sigma_{t}^{\varepsilon}$ as the solutions of the stochastic differential equations

$$
d \sigma_{t}^{\varepsilon}=\left(\frac{\kappa \theta}{2}-\frac{\nu^{2}}{8}\right) \Lambda_{\varepsilon}\left(\sigma_{t}^{\varepsilon}\right)-\frac{\kappa}{2} \sigma_{t}^{\varepsilon}+\frac{\nu}{2} d W_{t},
$$

with $\sigma_{0}^{\varepsilon}=\sigma_{0}$ for all $\varepsilon>0$.

Proposition 2.1. For each $t \in[0, T]$ the sequence $\sigma_{t}^{\varepsilon}$ converges to $\sigma_{t}$ in $L^{2}(\Omega)$.

Proof. We use the dominated convergence theorem in order to obtain this result. Let us first prove that $\sigma_{t}^{\varepsilon}$ converges to $\sigma_{t}$ point wise. This follows from a standard localization argument. For each $\varepsilon>0$ define a stopping time $\tau_{\varepsilon}$ via

$$
\tau_{\varepsilon}(\omega):=\inf \left\{t \mid \sigma_{t}(\omega) \leq \varepsilon\right\} .
$$

Letting $\varepsilon$ go to zero, the sequence of stopping times $\left(\tau_{\varepsilon}\right)$ defines an increasing sequence of stopping times, and it follows from equation (4) that $\lim _{\varepsilon \rightarrow 0} \tau_{\varepsilon}=$ $\infty$ a.s. Denoting with $\sigma^{\tau_{\varepsilon}}$ the process obtained from $\sigma$ by stopping at $\tau_{\varepsilon}$, then it follows from the choice of the function $\Lambda_{\varepsilon}(x)$ and equations (5) and (8), that

$$
\sigma_{t}^{\tau_{2 \varepsilon}}=\sigma_{t}^{\varepsilon} \quad \forall \quad t \leq \tau^{\varepsilon} .
$$

Now, for fixed $t \in[0, T]$ letting $\varepsilon$ go to zero one obtains that

$$
\lim _{\varepsilon \rightarrow 0} \sigma_{t}^{\varepsilon}=\sigma_{t} \text { a.s. }
$$

Let us now prove that for each $t \in[0, T] \sigma_{t}^{\varepsilon}$ converges to $\sigma_{t}$ in $L^{2}(\Omega)$. For this let us consider the Ornstein-Uhlenbeck process $u_{t}$ satisfying $u_{0}=\sigma_{0}$ and

$$
d u_{t}=-\frac{\kappa}{2} u_{t}+\frac{\nu}{2} d W_{t} .
$$

The Novikov condition as well as $0 \leq \Phi_{\epsilon}(x) \leq 1$ for all choices of $\varepsilon$ and $x$ imply that

$$
-\frac{\kappa}{2} \leq-\frac{\kappa}{2}+\left(\frac{\kappa \theta}{2}-\frac{\nu^{2}}{8}\right) \Lambda_{\varepsilon}(x) \leq-\frac{\kappa}{2}+\left(\frac{\kappa \theta}{2}-\frac{\nu^{2}}{8}\right) \frac{1}{x} .
$$

It therefore follows from Lemma 2.1 that

$$
u_{t} \leq \sigma_{t}^{\varepsilon} \leq \sigma_{t}
$$


and hence $\left|\sigma_{t}^{\varepsilon}\right| \leq\left|u_{t}\right|+\left|\sigma_{t}\right|$. The dominated convergence theorem implies the desired convergence.

Corollary 2.1. For each $t \in[0, T]$ the sequence $v_{t}^{\varepsilon}=\left(\sigma_{t}^{\varepsilon}\right)^{2}$ converges to the Heston volatility $v_{t}$ in $L^{1}(\Omega)$.

\section{A short review on Malliavin calculus}

Let us consider the cylindrical functional $F: \Omega \rightarrow \mathbb{R}$ given by

$$
F=f\left(W_{t_{1}}, \ldots, W_{t_{l}}\right)
$$

where $f \in C_{b}^{\infty}\left(\mathbb{R}^{l}\right)$ is a smooth function with bounded derivatives of all orders. Given $h \in L^{2}([0, T], \mathbb{R})$ we have that $\int_{0}^{\cdot} h(s) d s \in C_{0}([0, T], \mathbb{R})$ where the dot indicates that the upper bound of the integral is taken as a variable. The directional derivative of $F$ in direction $\int_{0}^{\cdot} h(s) d s$ at $\omega$ is given by

$$
\begin{aligned}
D_{h} F(\omega) & :=\left.\frac{d}{d \epsilon}\right|_{\epsilon=0} f\left(W_{t_{1}}(\omega)+\int_{0}^{t_{1}} h(s) d s, \ldots, W_{t_{l}}(\omega)+\int_{0}^{t_{l}} h(s) d s\right) \\
& =\sum_{i=1}^{m} \frac{\partial}{\partial x_{i}} f\left(W_{t_{1}}(\omega), \ldots, W_{t_{l}}(\omega)\right) \cdot \int_{0}^{t_{i}} h(s) d s .
\end{aligned}
$$

Now for fixed $\omega$ consider the linear bounded functional on $L^{2}([0, T])$ given by

$$
h \longmapsto D_{h} F(\omega) .
$$

By the Riesz-representation theorem there is an element $D F(\omega)$ in $L^{2}([0, T], \mathbb{R})$ such that

$$
D_{h} F(\omega)=\int_{0}^{T} D F(\omega)(s) h(s) d s, \forall h \in L^{2}([0, T]) .
$$

In the following we denote $D F(\omega)(s)$ with $D_{s} F(\omega)$. Let us now consider $\omega$ as a variable. The assumption that $f$ has bounded derivatives of all orders ensures that for all $p \geq 1$ we have $D F \in L^{p}\left(\Omega, L^{2}([0, T])\right)$ when considered as an $L^{2}([0, T])$ valued functional in $\omega$

Assume now that the functional $F$ is not necessarily cylindrical but there 
exists a sequence of cylindrical functionals $F_{i}$ such that $\left(F_{i}\right)$ converges to $F$ in $L^{p}(\Omega)$ and $\left(D F_{i}\right)$ converges to $G$ in $L^{p}\left(\Omega, L^{2}([0, T])\right)$. Then we define

$$
D F:=G=\lim _{i \rightarrow \infty} D F_{i} .
$$

The Cameron-Martin Theorem shows that this is indeed well defined and that the operator

$$
D: L^{p}(\Omega) \rightarrow L^{p}\left(\Omega, L^{2}([0, T])\right)
$$

defined on the cylindrical functionals is closable. The closure is called the Malliavin derivative operator and is denoted by $D$. The domain of $D$ is the closure of the cylindrical functionals under the norm

$$
\|F\|_{1, p}:=\|F\|_{L^{p}(\Omega)}+\|D F\|_{L^{p}\left(\Omega,\left(L^{2}[0, T]\right)\right)} .
$$

It is denoted with $\mathbb{D}^{1, p}$. In this article we concentrate on the case $p=2$. We will later make use of the following chain-rule ( see [19], Lemma 2.1 ) :

Proposition 3.1. Let $\phi: \mathbb{R} \rightarrow \mathbb{R}$ be a continuously differentiable function and $F \in \mathbb{D}_{1,2}$. Then $\phi(F) \in \mathbb{D}_{1,2}$ if and only if $\phi(F) \in L^{2}(\Omega)$ and $\phi^{\prime}(F) D F \in$ $L^{2}(\Omega \times[0, T])$ and in this case

$$
D_{t} \phi(F)=\phi^{\prime}(F) \cdot D_{t} F \text {. }
$$

The following formula for the Malliavin derivative of a diffusion is due to Detemple et al. [9]

Proposition 3.2. Consider a diffusion process $\left(X_{t}\right)$ satisfying the SDE

$$
d X_{t}=\mu\left(X_{t}\right)+\sigma\left(X_{t}\right) d W_{t}
$$

and assume that the coefficient functions satisfy $\mu \in C^{1}(\mathbb{R})$ and $\sigma \in C^{2}(\mathbb{R})$ as well as a linear growth condition and Lipschitz condition. Furthermore assume that $\sigma$ is positive and bounded away from zero. Then $X_{t} \in \mathbb{D}^{1,2}$ and for $\theta \leq t$ one has

$$
D_{\theta} X_{t}=\sigma\left(X_{t}\right) \exp \left(\int_{\theta}^{t} \mu^{\prime}\left(X_{s}\right)-\frac{\mu\left(X_{s}\right) \sigma^{\prime}\left(X_{s}\right)}{\sigma\left(X_{s}\right)}-\frac{1}{2} \sigma^{\prime \prime}\left(X_{s}\right) \sigma\left(X_{s}\right) d s\right)
$$




\section{The Malliavin derivative of the Heston volatil- ity}

In this section we will show that both the Heston volatility $v_{t}$ as well as it's square root $\sigma_{t}$ belong to $\mathbb{D}^{1,2}$. To do this we use our approximating sequence $\sigma_{t}^{\varepsilon}$ from section 2 . We have the following lemma :

Lemma 4.1. $\sigma_{t}^{\varepsilon} \in \mathbb{D}^{1,2}$ and $D_{\theta} \sigma_{t}^{\varepsilon}=\frac{\nu}{2} \exp \left\{\int_{\theta}^{t}\left[-\frac{\kappa}{2}+\left(\frac{\kappa m}{2}-\frac{\nu^{2}}{8}\right) \Lambda_{\varepsilon}^{\prime}\left(\sigma_{s}^{\varepsilon}\right)\right] d s\right\}$

Proof. This follows directly from Proposition 3.2.

We are now ready to proof our main result.

Theorem 4.1. We have $\sigma \in \mathbb{D}^{1,2}$ and $D_{\theta} \sigma_{t}=\frac{\nu}{2} \exp \left\{\int_{\theta}^{t}\left[-\frac{\kappa}{2}-\left(\frac{\kappa m}{2}-\frac{\nu^{2}}{8}\right) \frac{1}{\sigma_{t}^{2}}\right] d s\right\}$.

Proof. We know from Proposition 2.1 that for each $t \in[0, T]$ the sequence $\sigma_{t}^{\varepsilon}$ converges to $\sigma_{t}$ in $L^{2}(\Omega)$. Since this convergence is also point wise, we conclude by using the properties of the function $\Lambda_{\varepsilon}(x)$ that

$$
D_{\theta} \sigma_{t}^{\varepsilon}=\frac{\nu}{2} \exp \left\{\int_{\theta}^{t}\left[-\frac{\kappa}{2}+\left(\frac{\kappa \theta}{2}-\frac{\nu^{2}}{8}\right) \Lambda_{\varepsilon}^{\prime}\left(\sigma_{t}^{\varepsilon}\right)\right] d s\right\}
$$

converges point wise to

$$
G:=\frac{\nu}{2} \exp \left\{\int_{\theta}^{t}\left[-\frac{\kappa}{2}-\left(\frac{\kappa \theta}{2}-\frac{\nu^{2}}{8}\right) \frac{1}{\sigma_{t}^{2}}\right] d s\right\} .
$$

It follows from the Novikov condition, that the exponent in $D_{\theta} \sigma_{t}^{\varepsilon}$ is negative for all choices of $\varepsilon$ and therefore that $\left|D_{\theta} \sigma_{t}^{\varepsilon}\right| \leq \frac{\nu}{2}$ for all $\varepsilon$. It then follows from the bounded convergence theorem that $D_{\theta} \sigma_{t}^{\varepsilon}$ converges to $G$ in $L^{2}(\Omega)$. Finally it follows from Lemma 1.2 .3 in [18] that $\sigma_{t} \in \mathbb{D}^{1,2}$ and $D_{\theta} \sigma_{t}=G$.

Corollary 4.1. The Malliavin derivative of $\sigma_{t}$ is bounded and satisfies $\left|D_{\theta} \sigma_{t}^{\varepsilon}\right| \leq$ $\frac{\nu}{2}$. Furthermore $\sigma_{t} \in \mathbb{L}^{1,2}$.

Proof. This result follows from the proof above. It directly implies that $\sigma_{t} \in \mathbb{L}^{1,2}$ ( see Definition 1.3.2 ) in [18].

Corollary 4.2. The Heston volatility process $v_{t}$ is in $\mathbb{L}^{1,2}$ and

$$
D_{\theta} v_{t}=\nu \exp \left\{\int_{\theta}^{t}\left[-\frac{\kappa}{2}-\left(\frac{\kappa \theta}{2}-\frac{\nu^{2}}{8}\right) \frac{1}{v_{t}}\right] d s\right\} \sqrt{v_{t}}
$$


Proof. For fixed $t \in[0, T]$ we have $v_{t} \in L^{2}(\Omega)$ and

$$
\nu \exp \left\{\int_{\theta}^{t}\left[-\frac{\kappa}{2}-\left(\frac{\kappa \theta}{2}-\frac{\nu^{2}}{8}\right) \frac{1}{v_{t}}\right] d s\right\} \sqrt{v_{t}} \in L^{2}(\Omega) .
$$

It then follows from Lemma 2.1 in [19] that $v_{t} \in \mathbb{D}^{1,2}$. As in Corollary 4.1 one concludes from the explicit expression, that $\left(v_{t}\right) \in \mathbb{L}^{1,2}$.

Remark 4.1. Note that due to the Novikov condition, the expressions $\int_{\theta}^{t} \frac{1}{v_{t}} d s$ (and then $\int_{\theta}^{t}\left[-\frac{\kappa}{2}-\left(\frac{\kappa m}{2}-\frac{c^{2}}{8}\right) \frac{1}{v_{t}}\right] d s$ ) are well defined a.s.

\section{Conclusion}

We have proved that the Heston stochastic volatility or alternatively the CoxIngersoll-Ross process and their square roots are Malliavin differentiable and have given compact formulas for their derivatives. This is a key result in so far as that it opens the door for applications of Malliavin calculus in the framework of the Heston stochastic volatility model. The results underlines the role of the Heston stochastic volatility model, both under a theoretical and computational aspects.

\section{References}

[1] Alos, E. A Generalization of Hull and White Formula and Applications to Option Pricing Approximation (February 2004)

[2] Benhamou, E. Optimal Malliavin Weighting Function for the Computation of the Greeks. Mathematical Finance, Vol 13, Issue 1 (2003)

[3] Benth, F.-E.; Di Nunno, G.; Løkka, A.; Øksendal, B.; Proske, F. Explicit Representation of the Minimal Variance Portfolio in Markets Driven by Lévy Processes. Mathematical Finance, Vol 13, Issue 1 (2003)

[4] Bermin, H.-P. Hedging Options: The Malliavin Calculus Approach versus the $\Delta$-Hedging Approach. Mathematical Finance, Vol 13, Issue $1(2003)$

[5] Bouchard, B.; Ekeland I.; Touzi N. On the Malliavin approach to Monte Carlo approximation of conditional expectations. Finance and Stochastics 8 (2004) 
[6] Bouleau, N. Error Calculus and Path Sensitivity in Financial Models. Mathematical Finance, Vol 13, Issue 1 (2003)

[7] Cox, J.; Ingersoll, J. and Ross, S. A theory of the term structure of interest rates. Econometrica, 53: 385-408, (1985)

[8] Cvitanic,J.; Ma, J.; Zhang, J. Efficient Computation of Hedging Portfolios for Options with Discontinuous Payoffs. Mathematical Finance, Vol 13, Issue 1. (2003)

[9] Detemple, J.; Garcia, R.; Rindisbacher, M. Representation formulas for Malliavin derivatives of diffusion processes. Finance and Stochastics Volume 9, Number 3 (2005)

[10] Ewald, C.-O. Local Volatility in the Heston Model : A Malliavin Calculus Approach. Journal of Applied Mathematics and Stochastic Analysis, Vol. 2005, Issue 3, (2005)

[11] Ewald, C.-O. Optimal logarithmic Utility and optimal Portfolios for an Insider in a Stochastic Volatility Model. International Journal of Theoretical and Applied Finance, Vol. 8 No. 3 (2005)

[12] Ewald, C.-O.; Zhang, A. A new Technique for Calibrating Stochastic Volatility Models : The Malliavin Gradient Method. Submitted to Journal of Quantitative Finance (2005)

[13] Fournié, E.; Lasry, J-M.; Lebuchous, J.; Lions, P.-L., Touzi, N. Applications of Malliavin calculus to Monte Carlo methods in finance I. Finance Stoch. 3, No.4, 391-412 (1999).

[14] Fournié, E.; Lasry, J.-M.; Lebuchous, J.; Lions, P.-L. Applications of Malliavin calculus to Monte Carlo methods in finance II. Finance Stoch. 5, No.2, 201-236 (2001).

[15] Heston, S.L.; A Closed-Form Solution for Options with Stochastic Volatility with Applications to Bond and Currency Options; Review of Financial Studies, Vol.6, Issue 2 (1993)

[16] Imkeller, P. Malliavin's Calculus in Insider Models: Additional Utility and Free Lunches. Mathematical Finance, Vol 13, Issue 1 (2003)

[17] Karatzas, I.; Shreve, S.-E. Brownian motion and stochastic calculus. Graduate Texts in Mathematics, 113. New York etc. Springer-Verlag (1988) 
[18] Nualart, David The Malliavin calculus and related topics. Probability and Its Applications. Springer-Verlag (1995).

[19] Leon J.A., Navarro R., Nualart D.: An Anticipating Calculus Approach to the Utility Maximization of an Insider; Mathematical Finance, Vol 13, Issue 1 (2003) 